\title{
USEFULNESS OF PULSE PRESSURE VARIATION AS A TOOL TO ASSESS ADEQUACY OF INTRAVASCULAR VOLUME IN PATIENTS UNDERGOING ELECTIVE RENAL TRANSPLANTATION- AN OBSERVATIONAL STUDY
}

\author{
Bernice Theodore1, Tony Thomson Chandy²
}

${ }^{1}$ Assistant Professor, Department of Anaesthesiology, Christian Medical College Hospital, Vellore, Tamilnadu, India.

2Professor, Department of Anaesthesiology, Christian Medical College Hospital, Vellore, Tamilnadu, India.

\begin{abstract}
BACKGROUND
ABSTRACT

Pulse pressure variation is a dynamic parameter to assess intra-vascular volume and predict fluid responsiveness in mechanically ventilated patients. Its usefulness as an intra-operative monitoring tool in various situations is being studied. Chronic kidney disease is a growing health problem worldwide with the incidence of risk factors such as Diabetes Mellitus and Hypertension. A proportion of these patients will go on to develop end stage disease requiring renal replacement therapy either as maintenance dialysis or eventually renal transplantation. This study aims to assess the performance of pulse pressure variation as a tool to assess adequacy of intra-vascular volume in patients undergoing elective renal transplantation
\end{abstract}

\section{METHODS}

We studied 33 patients undergoing elective live related renal transplantation. All haemodynamic parameters including the heart rate, Arterial Blood Pressure, Central Venous Pressure (CVP) and Pulse Pressure Variation (PPV) were measured continuously throughout the procedure. Fluid therapy was carried out targeting a CVP of 10-15 mmHg. The time to urine output following revascularization of the renal graft (TUO) and urine volume in the first four hours were used as indirect indicators of adequacy of intravascular volume.

\section{RESULTS}

There was no linear correlation between Pulse Pressure Variation and Time to Urine output. However, based on ROC analysis, it was found that patients with a PPV of more than $10 \%$ would start producing urine only after 120 seconds of revascularization. There was however reasonable correlation between PPV and urine volume in the first 4 hours.

\section{CONCLUSIONS}

Pulse pressure variation could be used as a parameter to monitor the state of intravascular volume and aid in intra-operative fluid management of patients undergoing renal transplantation. A target PPV of $\leq 10 \%$ would be advisable to ensure immediate posttransplant diuresis

\section{KEY WORDS}

Pulse Pressure Variation, Intravascular Volume, Renal Transplantation, Post-Transplant Diuresis, Time to Urine Output

HOW TO CITE THIS ARTICLE: Theodore B, Chandy TT. Usefulness of pulse pressure variation as a tool to assess adequacy of intravascular volume in patients undergoing elective renal transplantation- an observational study. J. Evolution Med. Dent. Sci. 2019;8(25):1979-1982, DOI: 10.14260/jemds/2019/435

\section{BACKGROUND}

Chronic kidney disease is a growing health problem worldwide with the incidence of risk factors such as Diabetes Mellitus and Hypertension. A proportion of these patients will go on to develop end stage disease requiring renal replacement therapy either as maintenance dialysis or eventually renal transplantation.

Renal transplantation is considered successful if the end result is a healthy graft that functions optimally in the short and long term. Graft survival and function depends on multiple factors.(1) Graft perfusion is known to be a determinant of graft survival and function.

'Financial or Other Competing Interest': None.

Submission 18-04-2019, Peer Review 08-06-2019,

Acceptance 14-06-2019, Published 24-06-2019.

Corresponding Author:

Tony Thomson Chandy,

Professor,

Department of Anaesthesiology,

Christian Medical College Hospital,

Vellore, Tamilnadu, India.

E-mail: tonythomson@cmcvellore.ac.in

DOI: $10.14260 /$ jemds/2019/435
One of the key factors that determine graft perfusion is the adequacy of intravascular volume during the transplantation procedure.

Patients with end stage renal disease, awaiting renal transplant are usually on haemodialysis. Though perioperative dialysis corrects biochemical abnormalities and fluid overload, it results in intravascular volume depletion. It is important that this state of volume depletion is corrected as early as possible by administration of intravenous fluids to ensure adequate perfusion of the graft which in turn will determine its survival. Though a surrogate marker, graft diuresis is a reflection of adequacy of intra-vascular volume and also a predictor of function in the long term. Immediate urine output is the primary goal in live related renal transplantation. Delayed graft diuresis is found to decrease 1year survival rate from $75 \%$ to $49 \%$.(2)

The most widely used measure of intravascular volume is central venous pressure (CVP). CVP has been in use for many years and most anaesthesiologists are familiar with its use in goal directed fluid management.(3) Central venous pressure is a static measure of intravascular volume and not a true reflective of the dynamic nature of fluid volume shifts. 


\begin{tabular}{|c|c|c|}
\hline Parameter & Mean $(n=33)$ & Standard Deviation \\
\hline Age (years) & 32.18 & 10.9 \\
\hline Height $(\mathrm{cm})$ & 159.77 & 8.82 \\
\hline Pre-Dialysis weight (Kg) & 50.85 & 8.8 \\
\hline Post-Dialysis weight (Kg) & 49.4 & 7.96 \\
\hline Male & 22 & $66.67 \%$ \\
\hline Female & 11 & $33.3 \%$ \\
\hline \multicolumn{3}{|c|}{$\begin{array}{l}\text { Table 1. Demographic Data: Continuous Variables Expressed as } \\
\text { Mean and Standard Deviation, Sex Expressed as Percentage }\end{array}$} \\
\hline
\end{tabular}

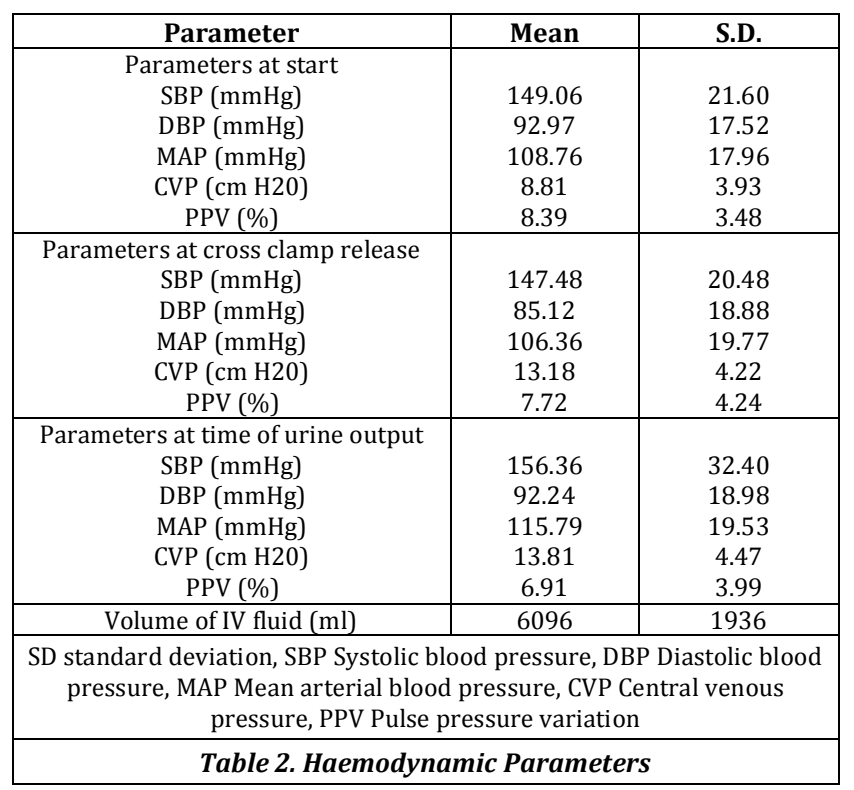
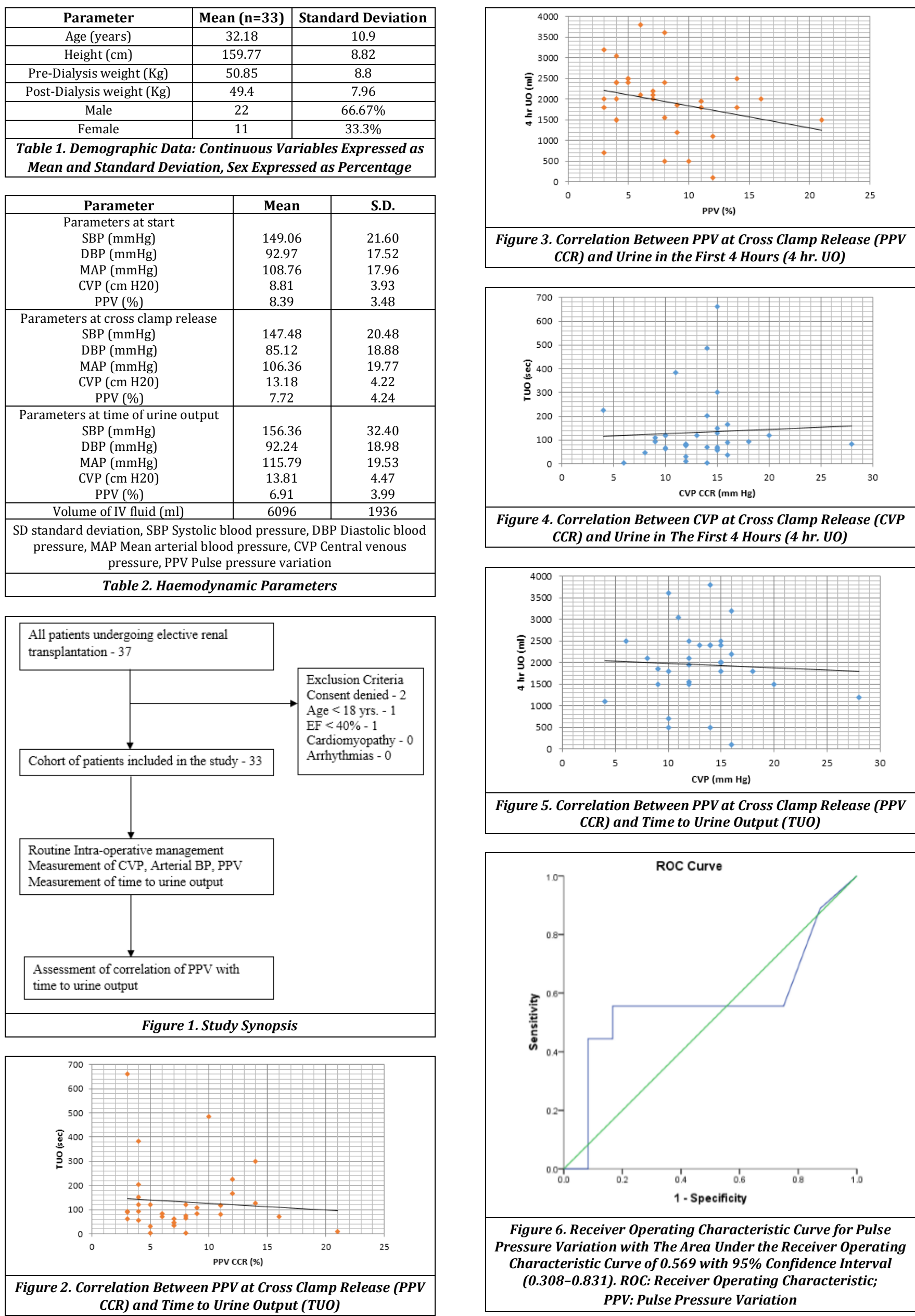

Figure 3. Correlation Between PPV at Cross Clamp Release (PPV CCR) and Urine in the First 4 Hours (4 hr. UO)

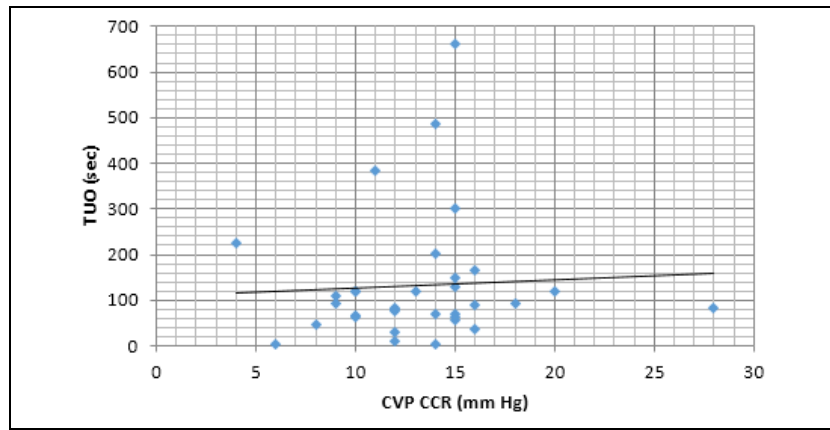

Figure 4. Correlation Between CVP at Cross Clamp Release (CVP CCR) and Urine in The First 4 Hours (4 hr. UO)

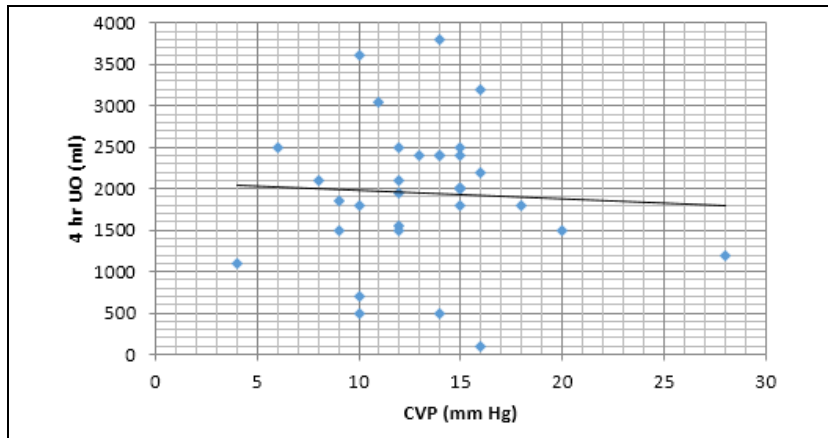

Figure 5. Correlation Between PPV at Cross Clamp Release (PPV CCR) and Time to Urine Output (TUO)

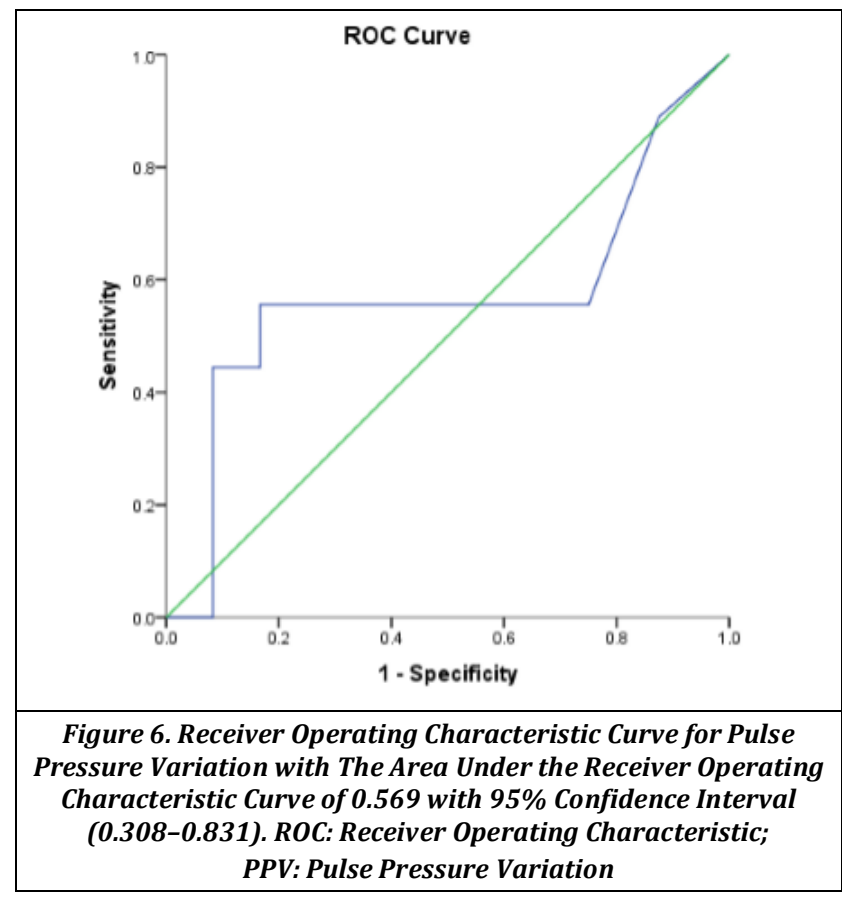


Newer parameters such as pulse pressure variation (PPV) and stroke volume variation (SVV) are dynamic parameters, have shown to be superior to CVP in assessing intravascular volume. $(4,5)$ Though it has become possible to measure these parameters real time with new anaesthetic monitors the accuracy of PPV has not been assessed adequately in various clinical scenarios. CVP remains the most widely used tool for measuring intravascular volume status during the intraoperative and early postoperative period. Often a CVP of $10-15 \mathrm{mmHg}$ is targeted for patients undergoing renal transplantation.(6) This study aims to assess the value of PPV in assessing intravascular volume as reflected by time to first urine output (TUO). The primary objective is to ascertain if there is a linear correlation between the PPV at the time of cross clamp release and TUO. The secondary objective was to find out the best cut off value of PPV that could be recommended for clinical use in patients undergoing renal transplantation so as to achieve adequate graft perfusion and function.

\section{METHODS}

Our study is a prospective observational study of a cohort design. Prior to commencing the study Institutional Research and Ethics Committee approval was obtained. All adult patients with end stage renal disease undergoing elective live donor renal transplantation were enrolled over a 10-month period. Written informed consent was obtained from the patients, the night before the surgery. We excluded patients younger than 18 years, with low cardiac function (ejection fraction < 40\%), cardiomyopathy, arrhythmias, and emergency renal transplants.

\section{Calculation of Sample Size}

In order to get a correlation of -0.8 (correlation between PPV and time to urine production) and show that it is significantly different from null hypothesis correlation of -0.5 , instead of 0 , keeping alpha and beta errors at 5\% and 20\% respectively a sample size of at least 29 was calculated.

\section{Intra-Operative Management Protocol}

In all patients, peripheral venous access was first established and basic monitoring (Electrocardiogram, Heart rate, Oxygen saturation and Non-invasive blood pressure) was commenced upon arrival to the operating room. Anaesthesia was induced with Fentanyl $2 \mathrm{mcg} / \mathrm{Kg}$, Propofol $2 \mathrm{mg} / \mathrm{Kg}$ and Rocuronium $0.1 \mathrm{mg} / \mathrm{Kg}$. Patients were intubated using an appropriate sized endotracheal tube and intermittent positive pressure ventilation established using a mechanical ventilator (Datex Ohmeda Aestiva/Aespire) with volume control ventilation and tidal volume set at $6-8 \mathrm{ml} / \mathrm{Kg}$. End tidal carbon dioxide monitoring was commenced after tracheal intubation. After induction, all patients had an ultrasound guided $8 \mathrm{~F}$ triple lumen catheter inserted into the internal jugular vein under aseptic precautions to measure central venous pressure (CVP) and a 20G cannula inserted into the radial artery on the hand without the arterio-venous fistula to monitor arterial blood pressure (ABP). Anaesthesia was maintained using isoflurane delivered in a mixture of oxygen and air. Paracetamol, Morphine and Fentanyl were used for intraoperative analgesia. Reversal of neuromuscular blockade was done with neostigmine and glycopyrrolate. Patients were extubated when fully awake.

\section{Haemodynamic Parameters}

All the vital parameters including the CVP and ABP were measured continuously throughout the procedure using a Philips MP50 Intellivue ${ }^{\mathrm{TM}}$ anaesthesia monitor. Intraoperative fluid administration was managed with balanced salt solutions and $0.9 \% \mathrm{NaCl}$ solutions so as to achieve a target a CVP more than $10-15 \mathrm{mmHg}$ at the time of reperfusion of the donor kidney. After vascular anastomosis was completed, blood flow to the graft was restored by release of the clamp on the vein and then the artery in that sequence. Hypotension, if noticed, was treated by either fluid boluses or vasopressors such as phenylephrine and ephedrine depending on the CVP, blood pressure and heart rate. The PPV value though monitored and recorded was not used to make decisions in fluid management. The time to urine output (in seconds) and volume of urine produced in the first 4 hours following revascularization of the renal graft (release of vascular clamps) were recorded and used as indirect indicators of adequacy of graft perfusion. The total estimated blood loss was noted down at the end of the operation.

\section{Statistical Methods}

The haemodynamic data were recorded and expressed as mean \pm standard deviation. Correlations between PPV and time to urine output, CVP and time to urine output, PPV and 4-hour urine output and CVP and 4-hour urine output were tested using the Pearson's correlation. Receiver operating characteristic (ROC) curve were generated for PPV and area under the ROC (AUROC) curve was calculated. All statistical analysis was carried out at $5 \%$ level of significance, and a $\mathrm{P}<$ 0.05 was considered significant. EPIDATA was used for data entry and statistical analysis.

\section{RESULTS}

Of the 37 elective renal transplantations that took place during the study period, 33 patients were included in the study. Two patients did not consent to participate, one was less than 18 years of age and one had severe cardiac disease with an ejection fraction of $40 \%$. There were 22 males and 11 females, and the mean age of the study group was 32.2 years. Most patients (22 of 33) were hypertensive. This was probably a consequence of the renal failure rather than its cause. Of the 33, 29 were on maintenance haemodialysis and one was on continuous ambulatory peritoneal dialysis. Three were not on any form of renal replacement therapy prior to transplant. The average duration between last haemodialysis and transplant for the 29 patients that were on haemodialysis was 19 hours.

It was observed that at the start of the operation only 16 of the $33(48.48 \%)$ patients had CVP that was within the desired range of $10-15 \mathrm{mmHg}$. At the time of reperfusion, 28 of the $33(84.84 \%)$ patients had a CVP within or above the desired range of 10-15 mmHg. By this it could be inferred that a significant number of the patients were in a fluid depleted state at the start of the operation, but with intraoperative fluid management this state was corrected in most of them by the time the renal graft was re-perfused. The haemodynamic parameters are summarized in Table 2 .

It was observed that 24 of the 33 patients $(72.7 \%)$ had a urine output within 120 seconds of revascularization of the graft and most of patients had a urine output of $>1 \mathrm{ml} / \mathrm{Kg}$ body weight in the first 4 hours after revascularization. 
There was no correlation observed between PPV and TUO (Figure 2). There was correlation noted between PPV and urine output measured in the first 4 hours (Figure 3). However this was weak, the correlation coefficient being $(-0.273)$. The sensitivities and specificities for different values of PPV were plotted on a curve and urine output within 120 seconds of revascularization of the graft used as the desired value for ROC analysis (Figure 6). ROC analysis revealed that PPV value of $10 \%$ could be used as a cut off for clinical use as for patients whose PPV value was $>10 \%, 83.33 \%$ had a time to urine output of more than 120 seconds. There was no correlation observed between CVP and both TUO and urine output measured in the first 4 hours (Figures 4,5 ).

\section{DISCUSSION}

Outcome following renal transplantation is dependent on many factors. Some of these are immunological and therefore not under the control of the surgical procedure. Also, donor kidney related factors have a role to play in graft function and outcomes. There are technical or mechanical factors often directly related to the actual surgical procedure which have over the years been standardized. Of the technical factors, the one factor that is directly under the control of the anaesthesiologist is the intravascular volume status of the recipient. This in turn has a bearing on the cardiac output and therefore perfusion of the graft. The intravascular volume status is controlled effectively by fluid management.

Fluid management in renal transplant can be specially challenging to the anaesthesiologist as most patients are in a state of fluid depletion as a result of haemodialysis while waiting for a transplant. There is a need to correct this fluid deficit before the time of perfusion of the renal graft $(7,8)$ that occurs when vascular cross clamps on the vascular pedicle of the graft is released. To ensure appropriate fluid management, various haemodynamic parameters are measured during the course of the operation. The most widely used parameter to assess intravascular volume is central venous pressure (CVP). Though this has been used for many years and is widely accepted by most anaesthesiologists and intensivists as a good indicator of intravascular volume, it has its limitations. Primarily it is based on the assumptions about the relationship between the pulmonary and systemic circulations and that pressure in the cardiac chambers reflects volume. This may not always be the case, particularly in patients undergoing intermittent positive pressure ventilation with positive end expiratory pressure. Renal transplants are invariably done under general anaesthesia and patients are on IPPV with PEEP. Secondly the use of central venous pressure may not be reliable when the patient's position is changed during the operation. This too happens frequently during the course of the operation to facilitate better vision during the vascular anastomosis.

The primary advantage of PPV is that being a dynamic parameter it is unaffected by variations of intrathoracic pressure and patient position. It is recorded from a peripheral arterial canula and therefore does not require central venous access that may be associated with line sepsis considering the renal graft recipient is under immunosuppression. Direct arterial pressure monitoring is routinely done during renal transplant surgery and with newer anaesthetic monitors with the facility to measure PPV, this parameter can be easily monitored in all patients.
The aim of this study was to ascertain if there was a correlation between PPV and intravascular volume as measured indirectly by the time between reperfusion of the graft and onset of post-transplant diuresis and the volume of urine produced in the first four hours.

It was found that neither CVP nor PPV had a correlation with time to urine output at the time of reperfusion of the renal graft. However, there was weak correlation between PPV and urine volume in the first four hours post transplantation.

However, the ROC curve analysis revealed that if a certain target PPV could be achieved at the time of reperfusion of the renal graft, this was a reasonably good predictor of time to urine output as the specificity was as high as $85 \%$ though the sensitivity was not higher than $50 \%$. If the PPV is $>10 \%$, there was a high likelihood of the time to urine output being more than 120 seconds. Therefore, it was inferred that the PPV value to target would be $\leq 10 \%$.

\section{CONCLUSIONS}

Pulse pressure variation could be used as a parameter to monitor the state of intravascular volume and aid in intraoperative fluid management of patients undergoing renal transplantation. A target PPV of $\leq 10 \%$ would be advisable to ensure immediate post-transplant diuresis.

\section{REFERENCES}

[1] Ferris RL, Kittur DS, Wilasrusmee C, et al. Early haemodynamic changes after renal transplantation: determinants of low central venous pressure in the recipients and correlation with acute renal dysfunction. Med Sci Monit 2003;9(2):CR61-6.

[2] Sprung J, Kapural L, Bourke DL, et al. Anesthesia for kidney transplant surgery. Anesthesiology Clinics of North America 2000;18(4):919-51.

[3] Magder S, Erice F, Lagonidis D. Determinants of the $Y$ descent and its usefulness as a predictor of ventricular filling. J Intensive Care Med 2000;15(5):262-9.

[4] Kramer A, Zygun D, Hawes H, et al. Pulse pressure variation predicts fluid responsiveness following coronary artery bypass surgery. Chest 2004;126(5):1563-8.

[5] Benes J, Chytra I, Altmann P, et al. Intraoperative fluid optimization using stroke volume variation in high risk surgical patients: results of prospective randomized study. Crit Care 2010;14(3):R118.

[6] Lemmens HJ. Kidney transplantation: recent developments and recommendations for anesthetic management. Anesthesiol Clin North America 2004;22(4):651-62.

[7] Rang ST, West NL, Howard J, et al. Anaesthesia for chronic renal disease and renal transplantation. European Association of Urology and European Board of Urology Update 2006:246-56. [Series 4].

[8] Othman MM, Ismael AZ, Hammouda GE. The impact of timing of maximal crystalloid hydration on early graft function during kidney transplantation. Anaesthesia \& Analgesia 2010;110(5):1440-6. 